\title{
Association of esophageal dilatation in chest CT scan with gastroesophageal reflux disease: A case control study
}

\author{
Seyed Jalal Hashemi1, Seyed Ali Javad Moosavi², Esmaeil Idani³, Ali Javaherfuroushzadeh4, \\ Hanieh Raj3
}

\author{
1 Alimentary Tract Research Center (ATRC), Ahvaz Jundishapur University of Medical Sciences \\ and Health Services, Ahvaz
}

2 Air Pollution Research Center, Iran University of Medical Sciences and Health Services, Tehran

3 Air Pollution and Respiratory Diseases Research Center, Ahvaz Jundishapur of Medical Sciences and Health Services, Ahvaz

4 Department of Internal Medicine, Ahvaz Jundishapur University of Medical Sciences and Health Services, Ahvaz, Iran

\begin{abstract}
The presence of increased air bubble in the lumina of esophagus on a chest CT scan may be associated with esophageal disorders. The purpose of this study was to determine the association between the appearance of air bubbles on chest CT scan and gastroesophageal Reflux Disease (GERD). In this case-control study, thirty-two patients with endoscopically proved GERD and 32 subjects without GERD underwent
\end{abstract}

Corresponding author: Hanieh Raji, Air Pollution and Respiratory Diseases Research Center, Ahvaz Jundishapur University of Medical Sciences, Ahvaz, Iran.

Tel.Fax +98.61.32216104. E-mail: dr.raji.h@gmail.com

Key words: Esophageal diseases; gastroesophageal reflux; computed tomography; chronic cough.

Contributions: $\mathrm{SJH}$, data collection, interpretation and analyses, manuscript drafting; SAJM, EI, study design, analyses and interpretation of data; AJ, data collection, analyses and interpretation of data; HR, study design, data collection, analyses and interpretation, manuscript drafting. All authors read and approved the final manuscript.

Conflict of interest: The authors declare no competing interests.

Funding: This study was funded by Alimentary Tract Research Center (ATRC), Ahvaz Jundishapur University of Medical Sciences and Health Services.

Acknowledgments: The trial protocol was approved by the Institutional Ethical Review Board at Ahvaz Jundishapur University of Medical Sciences (AJUMS). The cooperation of all participants in this study is greatly appreciated.

Received for publication: 23 February 2018

Accepted for publication: 21 May 2018

(C) Copyright S.J. Hashemi et al., 2018

Tipografia PI-ME Editrice, Italy

Monaldi Archives for Chest Disease 2018; 88:914

doi: 10.4081/monaldi.2018.914

This article is distributed under the terms of the Creative Commons Attribution Noncommercial License (by-nc 4.0) which permits any noncommercial use, distribution, and reproduction in any medium, provided the original author(s) and source are credited. chest computed tomography (CT) scanning. Esophageal dilatation (ED) was defined as the presence of air bubbles greater than $10 \mathrm{~mm}$ in the supra ventricle (SV) and ventricle (CV), and air bubbles $>15 \mathrm{~mm}$ in the ventricle to the lower esophageal sphincter (V-LES). The results were compared between the two groups. The GERD patients included $16(50 \%)$ males with a mean age of $58.5 \pm 11.2$ years and the control group included 17 (54.8\%) males and 14 (45.1\%) females with a mean age of $66.7 \pm 10.5$ years. There was a significant relationship between the presence of GERD and esophageal dilation (ED) in the V-LES sections on the CT scan $(\mathrm{P}=0.002)$. The mean size of the air bubbles in the V-LES section was $11.73 \mathrm{~mm}$ in the case group in comparison to 4.32 $\mathrm{mm}$ in the control group $(\mathrm{P}<0.001)$. The size and location of the air bubbles in the esophagus can vary and be important. The possibility of GERD increases in the presence of esophageal dilation on CT scan.

\section{Introduction}

Gastroesophageal reflux disease (GERD) is one of the most common gastrointestinal (GI) diseases that creates a major impact on the quality of life and imposes high burdens and financial costs on the world population [1,2]. It affects $10-20 \%$ of the western and $20 \%$ of the Iranian population [3]. Heartburn is the most common symptom of GERD but a broad spectrum of extra-esophageal manifestations, including laryngitis, dental erosions, hoarseness, chronic cough, noncardiac chest pain, recurrent pneumonia, asthma, subglottic stenosis, and laryngeal cancer have been reported [4]. Esophageal computed tomography (CT) scan is not the standard of care for the evaluation of GERD, but it is an essential modality for respiratory disorders. The presence of air bubbles on chest CT scan of such patients is a matter of debate. The air is commonly detected on radiological exams of the normal esophagus. It has been reported that an air column is visible in $64 \%$ of normal esophagus CT scans [5]. Esophageal dilatation (ED) was determined as the presence of an air column greater than $10 \mathrm{~mm}$ in the coronal plane [6]; the definition then changed to the presence of air bubbles greater than $10 \mathrm{~mm}$ in the supra ventricle (SV) and ventricle (CV), and air bubbles $>15 \mathrm{~mm}$ in the ventricle to the lower esophageal sphincter (V-LES) [7]. The clinical significance of incidental esophageal dilatation seen on the chest CT scan of a patient without any GI symptoms is challenging. In a recent study, the appearance of esophageal air bubbles on the CT scan was associated with an increased frequency of GERD symptoms [8]. Respiratory disorders including asthma and pulmonary fibrosis have been reported as a com- 
plication of GERD; on the other hand, ED is a well-known entity in some systemic disorders that affect respiratory and GI systems. The presence of ED on the chest CT scan may be a sign of esophageal or systemic disorders such as GERD and systemic sclerosis [9]. In the present study, we evaluated the association between endoscopy proven GERD and ED or the size and location of air bubbles on esophageal CT images.

\section{Materials and Methods}

\section{Study population}

We carried out this case-controlled study in Imam Khomeini Hospital affiliated with Ahvaz Jundishapur University of Medical Sciences (AJUMS) between December 2012 and May 2014. A total of 32 GERD patients that were diagnosed by upper GI endoscopy were enrolled in a prospectively. The control group comprised patients without GERD and GI symptoms who were admitted to the pulmonary ward. All patients and controls underwent chest CT scan. Due to the association between hiatal hernia and GERD, the patients who had hiatal hernia on upper GI endoscopy or CT scan were excluded from the study. The CT images were reviewed by a trained pulmonologist who was not aware of the GERD status of the patients. The presence of the air was characterized by its location, quantity, diameter on the CT scan, and the presence or absence of ED. Informed consent was obtained from the case group. Ethics Committee of AJUMS approved this study.

\section{Radiologic measurement}

The patients were scanned at full inspiration using a Siemens scanner with 1- to 2-mm collimation. The images were reconstructed to a 512-pixel matrix using a sharp kernel and a 26 -cm display field of view. They were transferred to an image analysis workstation. Just standard mediastinal windows (width, $450 \mathrm{HU}$; level, $50 \mathrm{HU}$ ) and paranchymal windows (width, $1300 \mathrm{HU}$; level, -290 HU) were viewed. We used the guidelines given by Schraufnagel; the measurements were all limited to the thoracic esophagus. The presence of air in the esophagus and esophageal dilatation (ED) were scrutinized.

In each transvers section the largest linear measurement of the esophageal air column was measured until the esophagus adhered to the stomach, which was usually in the first abdominal CT section. If the esophagus was folded and included two air densities, we measured only the largest one because the measurement line was restricted to the air only. The internal measurements were recorded for each section. The esophagus was divided into 3 sections, including the supra ventricle (SV), ventricle (CV) and ventricle to lower esophageal sphincter (V-LES) and the normal air column was assessed in different parts of the esophagus by CT scan. ED was defined as the presence of air bubbles $>10 \mathrm{~mm}$ in the SV and CV, and greater than $15 \mathrm{~mm}$ in the V-LES (Figure 1 A,B).

\section{Statistical analysis}

SPSS software version 16 (Chicago, IL, USA) was used for statistical analysis. The data is expressed as mean $\pm \mathrm{SD}$. independent $\mathrm{T}$ test was used to compare all continuous variables and categorical variables were compared with Pearson chi-square test or Fisher's exact test. The difference between grade A, B, and C in ED was assessed by the analysis of covariance. Significant p-values were considered less than 0.05 .

\section{Results}

A total of 32 GERD patients with a mean age of $58.5 \pm 11.2$ years including 16 (50\%) males and16 (50\%) females were enrolled in this study. In the GERD group, 4 patients had hiatal hernia and according to Los Angeles (LA) classification of esophagitis, 46.8\% (95\% CI: 29.0965.25) had esophagitis grade A, 50\% (95\% CI 31.88-68.11) had esophagitis grade B, and $4.2 \%$ (95\% CI: $0.7-20.8)$ had esophagitis grade $\mathrm{C}$. There was no significant correlation between age and the grade of esophagitis ( $\mathrm{p}=0.447)$.

Thirty-one subjects without GERD with a mean age of $66.7 \pm 10.5$ years, including 17 (54.8\%) males and 14 (45.1\%) females were enrolled in the control group. There was no significant difference in female and male gender between the two groups ( $p=0.208)$. ED in the V-LES section was seen in 15 (46.9\%) GERD patients and 3 (9.7\%) individuals in the control group subsequently $(\mathrm{p}=0.02)$ (Table 1). The mean size of the esophageal air bubble in the V-LES section was significantly larger in the GERD group than the control group $(11.73 \mathrm{~mm}$ versus $4.32 \mathrm{~mm}, \mathrm{p}<0.001$ ) (Table 2). Exclusion of cases with hiatal
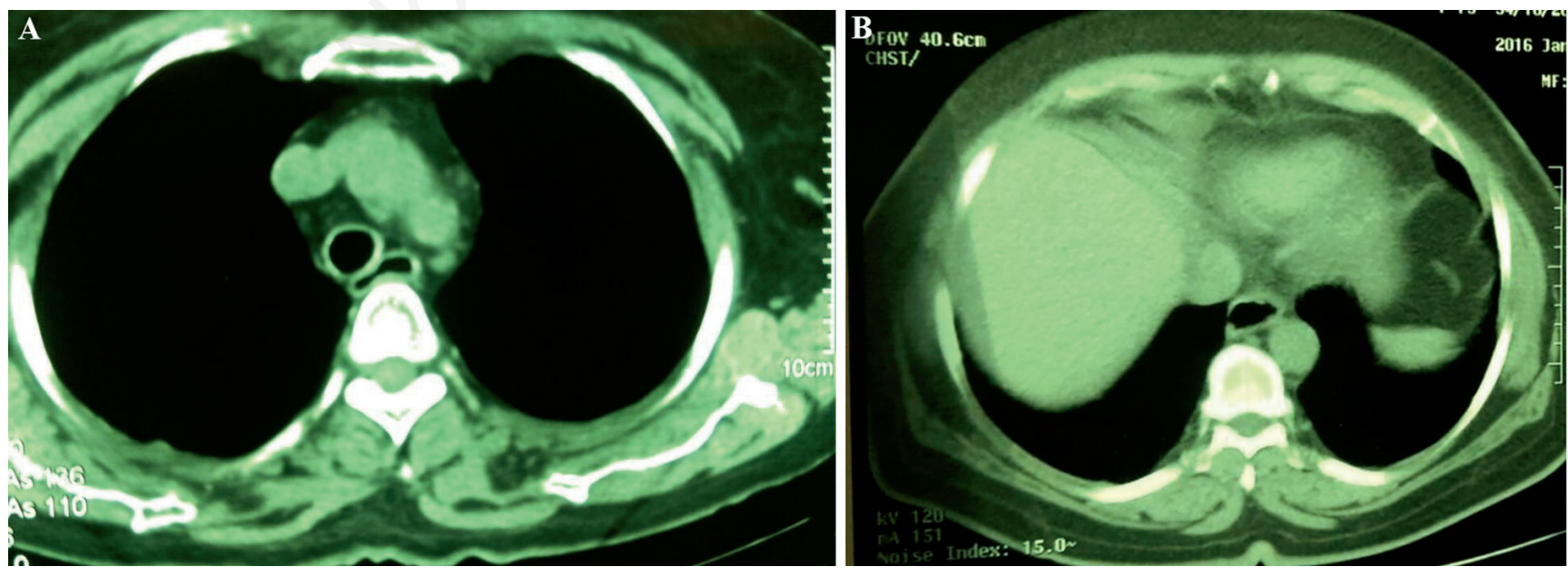

Figure 1. A) CT scan in 52-year-old man, with esophageal dilatation in supra ventricular section; the greatest direct distance of air bubble of esophagus was measured; the soft tissue was not included in the recording line; scale is in millimeters. B) Lumen of air bubble at V_LES section in the same patient. 
hernia (reported on CT scan or upper GI endoscopy) did not change the results after data reanalysis. There were no significant differences in the mean size of esophageal air bubbles in SV and CV sections between the two groups. There is a decreasing measure of the ED rate from the upper esophageal section to the middle and distal sections in the con- trol group (51.6\%, 22.6\%, and 9.7\%, respectively). But the rate of ED in the distal section of the esophagus was higher than the middle section in the GERD group (Figure 2). There was no significant difference between esophageal dilation and the grade of esophagitis by covariance analysis (Table $s 3$ and 4).

Table 1. Comparison of the frequency (\%) air bubble ( $\mathrm{mm})$ size in case and control group.

\begin{tabular}{lcccc} 
& & Case & Control & p-value \\
SV $(\mathrm{mm})$ & $\geq 10$ & $21(65.6 \%)$ & $16(51.6 \%)$ & 0.3 \\
\hline CV $(\mathrm{mm})$ & $<10$ & $11(34.4 \%)$ & $15(48.4 \%)$ & 0.4 \\
\hline & $\geq 10$ & $11(34.4 \%)$ & $7(22.6 \%)$ & \\
\hline V_LES $(\mathrm{mm})$ & $<10$ & $21(65.6 \%)$ & $24(77.4 \%)$ & 0.002 \\
\hline & $\geq 15$ & $15(46.9 \%)$ & $3(9.7 \%)$ & \\
\hline
\end{tabular}

$\mathrm{SV}$, supra ventricle; $\mathrm{CV}$, cardiac ventricle; $\mathrm{V} \_\mathrm{LES}$, ventricle to lower esophageal sphincter.

Table 2. Comparison of the air bubble $(\mathrm{mm})$ size in case and control group.

\begin{tabular}{lcccc} 
& Patients $n=32$ & Controls $n=31$ & p-value & $95 \%$ CI \\
SV $($ mean \pm SD) & $9.15 \pm 6.6$ & $9.09 \pm 8.5$ & 0.9 & $-3.7-3.9$ \\
\hline CV & $5.68 \pm 7.6$ & $3.72 \pm 6.1$ & 0.2 & $-1.5-5.4$ \\
\hline V_LES & $11.73 \pm 6.67$ & $4.32 \pm 6.2$ & $<0.001$ & $4.5-10.6$
\end{tabular}

$\mathrm{SV}$, supra ventricle; CV, cardiac ventricle; $\mathrm{V} \_$LES, ventricle to lower esophageal sphincter.

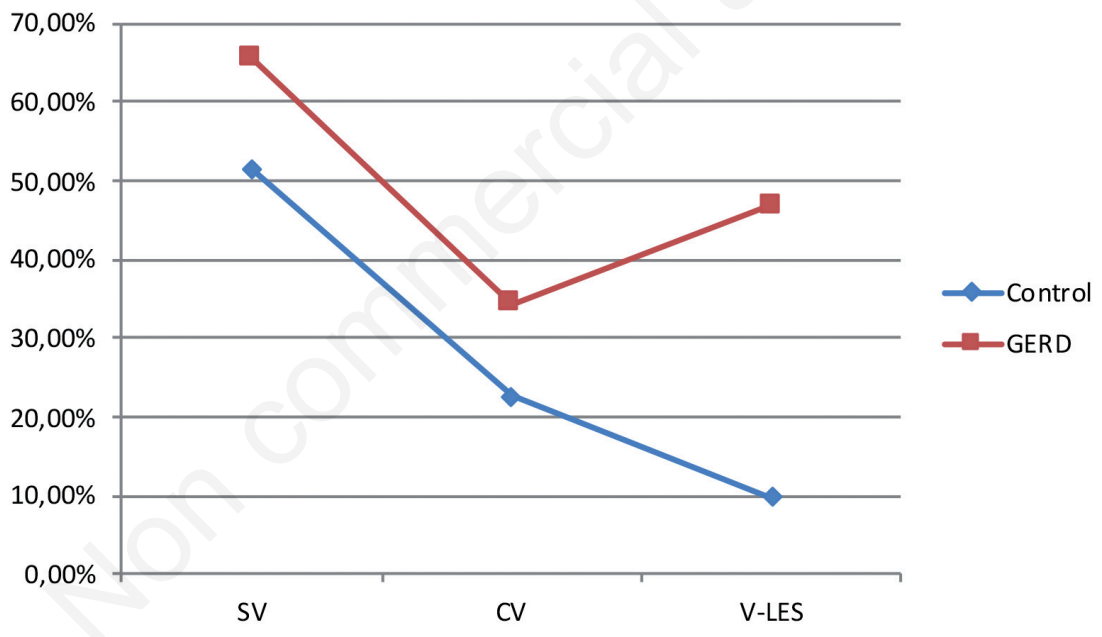

Figure 2. Rate of esophageal dilatation at different level of esophagus.

Table 3. Comparison air bubble ( $\mathrm{mm}$ ) size in V_LES section due to GERD staging.

\begin{tabular}{lccccc} 
& \multicolumn{3}{c}{ GERD } & C & \\
\hline V_LES & & A & B & $1(5.9 \%)$ & \\
\hline & $<15 \mathrm{~mm}$ & $8(47.1 \%)$ & $8(47.1 \%)$ & $1(6.7 \%)$ & 0.9 \\
\hline
\end{tabular}

V_LES, ventricle to lower esophageal sphincter

Table 4. Comparison between air bubble ( $\mathrm{mm})$ size due to GERD severity.

\begin{tabular}{lccccc} 
& GERD A & GERD B & GERD C & & \\
SV $(\mathrm{mm})$ & $8.9 \pm 6.2$ & $9.2 \pm 6.5$ & $10.0 \pm 14.0$ & 0.9 & 0.7 \\
\hline CV $(\mathrm{mm})$ & $5.3 \pm 7.9$ & $5.4 \pm 7.0$ & $10.0 \pm 14.0$ & $15.0 \pm 7.07$ & 0.7 \\
\hline V-LES $(\mathrm{mm})$ & $11.0 \pm 6.5$ & $11.8 \pm 7.04$ & 1 & \\
\hline
\end{tabular}

$\mathrm{SV}$, supra ventricle; $\mathrm{CV}$, cardiac ventricle; $\mathrm{V} \_$LES, ventricle to lower esophageal sphincter. 


\section{Discussions}

The presence of air bubbles in the esophagus is frequently detected on radiological examination of at least $60 \%$ of normal patients with various sizes and locations. Less than $3 \%$ of the subjects have air in the lower two sections, that indicates the normal lower esophageal sphincter is closed. According to a study by Schraufnagel [7], distension of the esophagus up to $15 \mathrm{~mm}$ should be considered normal in the position below the ventricles until above the diaphragm or V-LES, but any measurement of $20 \mathrm{~mm}$ or more should be considered abnormal. The presence of increased esophageal air bubbles or dilation of the esophageal lumen on a chest CT scan may be correlated with esophageal disease and may be a clue for diagnosing states such as scleroderma, esophageal cancer, achalasia, or other benign distal esophageal strictures [6,10-13]. Most of diseases that result in esophageal dilatation present with dysphagia or regurgitation but respiratory symptoms may be the essential complains of patients with GERD.

For the first time, Moosavi et al. evaluated the association between the presence of air bubbles on thoracic CT scan and GERD symptoms in a cross-sectional study; they showed a significant relationship between the presence of ED in the V-LES and heartburn (76.9\% versus $32.3 \%$ in patients with and without ED) [8]. That study was cross-sectional and included patients with major and minor GERD symptoms via a checklist and had no control group, so we performed this case- control study on endoscopically proven GERD patients to address this correlation.

In the present study, we found a correlation between the presence of air bubbles at the V-LES sections on CT scan and GERD. Esophageal dilatation was observed in about $47 \%$ of GERD and $10 \%$ of control subjects $(\mathrm{p}=0.02)$ (Table 1). The mean size of the esophageal air bubbles in the V-LES section was significantly larger in the GERD versus the control subjects. There were no significant differences in the mean size of esophageal air bubbles in the upper esophageal sections between the two groups (Table 2). We realized a decreasing measure of the ED rate from the upper esophageal section to the middle and distal sections in the control group (51.6\%, 22.6\%, and 9.7\%, respectively). The rate of ED in the distal section of the esophagus was higher than the middle section in the GERD group (Figure 2). This finding is compatible with the observation of Schraufnagel [7], indicating that air bubbles in the upper esophageal sections may be secondary to air swallowing whereas increased air bubbles in the distal sections are related to GERD.

Esophageal dilatation in the V-LES section might be a consequence of or transient lower esophageal relaxation (TLESR) or lower esophageal sphincter (LES) dysfunction. TLESR is an important issue in the pathophysiology of GERD [14,15]. Sifrim et al. showed that in mixed reflux events, liquid often precedes gas reflux. They observed that gas reflux occurred as the primary event in only a minority of acid reflux episodes [16]. Mixed reflux of liquid and gas is more frequent than pure liquid reflux, both in healthy subjects and in GERD patients. Bredenoord and Weusten reported a significant correlation between gas reflux and air swallowing. Air swallowing did not provoke the reflux of the liquid or mixed gas-liquid gastric contents in healthy volunteers. They found no correlation between the size of the gastric air bubble and different reflux parameters [17].

In spite of recent advances in continuous imaging with 320 row area detector CT scan as described by Fukazawa et al. [18], and positron emission tomography/computed tomography (PET/CT) as reported by Wu et al. [19] for gastrointestinal disorders, these imaging modalities are not standard of care for diagnosis of GERD, so detection of ED on chest CT scan or high-resolution chest CT scan (HRCT ) is an incidental and challenging finding. Firstly, esophageal stasis may predis- pose the patient to the recurrent microaspiration and different respiratory complications including lung abscess, asthma and interstitial lung disease (ILD) [1,20-23]. Secondly, ED might be correlated with the severity of lung disease or may be a clue for diagnosis of a serious underlying disorder. Carrie Richardson et al. in a cross- sectional study on 270 cases of systemic sclerosis found that esophageal dilatation on HRCT is correlated with more sever ILD. Affected patients had more sever imaging and functional abnormality including lower lung volume and lower DCLO predicted [21]. Since esophageal motility disorder and TLESR are common in GERD, it is reasonable to see esophageal dilatation with its respiratory complications [24].

Our results suggest that CT scan could be potentially useful in detecting thoracic esophageal dilatation in patients who have GERD; whoever, our study did not support any correlation between ED and the severity of GERD. This study is the first prospective research showing a correlation between air bubbles on CT scan and GERD.

Our study has certain limitation; this study was designed to evaluate reflux esophagitis and patients with non-erosive reflux disease (NERD) were not included; therefore, further studies using $24 \mathrm{~h} \mathrm{pH}$ monitoring as gold standard of GERD diagnosis may be helpful.

\section{Conclusions}

In conclusion, our findings demonstrated that esophageal dilatation and increased air bubbles in the esophageal lumen in the V-LES sections of chest CT scan were more common in patients with esophagitis than normal individuals. We found no relationship between the size of the esophageal air column and the severity of esophagitis.

\section{References}

1. Goldman J, Bennett JR. Gastro-oesophageal reflux and respiratory disorders in adults. Lancet 1988;2:493-5.

2. Wiklund I. Review of the quality of life and burden of illness in gastroesophageal reflux disease. Dig Dis 2004;22:108-14.

3. Delavari A, et al. Gastroesophageal reflux disease burden in Iran. Arch Iran Med 2015;18:85-8.

4. Locke GR 3rd, Talley NJ, Fett SL, et al. Prevalence and clinical spectrum of gastroesophageal reflux: a population-based study in Olmsted County, Minnesota. Gastroenterology 1997;112:1448-56.

5. Halber MD, Daffner RH, Thompson WM. CT of the esophagus: I. Normal appearance. AJR Am J Roentgenol 1979;133:1047-50.

6. Bhalla M, Silver RM, Shepard JA, McLoud TC. Chest CT in patients with scleroderma: prevalence of asymptomatic esophageal dilatation and mediastinal lymphadenopathy. Am J Roentgenol 1993;161:269-72.

7. Schraufnagel DE, Michel JC, Sheppard TJ, et al. CT of the normal esophagus to define the normal air column and its extent and distribution. Am J Roentgenol 2008;191:748-52.

8. Moosavi A, Raji H, Teimoori M, et al. Air column in esophagus and symptoms of gastroesophageal reflux disease. BMC Med Imaging 2012;12:2.

9. Vonk MC, van Die CE, Snoeren MM, et al. Oesophageal dilatation on high-resolution computed tomography scan of the lungs as a sign of scleroderma. Ann Rheum Dis 2008;67:1317-21.

10. Pandey AK, Wilcox P, O' Brien J, et al. Significance of various pulmonary and extrapulmonary abnormalities on HRCT of the chest in scleroderma lung. Indian J Radiol Imaging 2013;23:304-7.

11. Sugiura T, Iwakiri K, Kotoyori M, Kobayashi M. Relationship between severity of reflux esophagitis according to the Los Angeles classification and esophageal motility. J Gastroenterol 2001;36:226-30. 
12. Moss WJ, Pang J, Orosco RK, et al. Esophageal dilation in head and neck cancer patients: A systematic review and meta-analysis. Laryngoscope 2018;128:111-7.

13. O'Neill OM, Johnston BT, Coleman HG. Achalasia: a review of clinical diagnosis, epidemiology, treatment and outcomes. World J Gastroenterol 2013;19:5806-12.

14. Blackshaw LA, Staunton E, Lehmann A, Dent J. Inhibition of transient LES relaxations and reflux in ferrets by GABA receptor agonists. Am J Physiol 1999;277:G867-74.

15. Kawahara H, Dent J, Davidson G. Swallowing and LES relaxation with reflux: not by chance. Gastroenterology 1998;115:515.

16. Sifrim D, Castell D, Dent J, Kahrilas PJ. Gastro-oesophageal reflux monitoring: review and consensus report on detection and definitions of acid, non-acid, and gas reflux. Gut 2004;53:1024-31.

17. Bredenoord AJ, Pandolfino JE, Smout AJ. Gastro-oesophageal reflux disease. Lancet 2013;381:1933-42.

18. Fukazawa K, Furuta K, Adachi K, et al. Continuous imaging of esophagogastric junction in patients with reflux esophagitis using 320-row area detector CT: a feasibility study. J Gastroenterol Hepatol 2013;28:1600-7.
19. Wu YW, Tseng PH, Lee YC, et al. Association of esophageal inflammation, obesity and gastroesophageal reflux disease: from FDG PET/CT perspective. PloS One 2014;9:e92001.

20. Zhang $\mathrm{C}, \mathrm{Wu} \mathrm{J}, \mathrm{Hu} \mathrm{Z}$, et al. Diagnosis and anti-reflux therapy for GERD with respiratory symptoms: A study using multichannel intraluminal impedance-pH monitoring. PloS One 2016;11:e0160139.

21. Richardson C, Agrawal R, Lee J, et al. Esophageal dilatation and interstitial lung disease in systemic sclerosis: A cross-sectional study. Semin Arthritis Rheum 2016;46:109-14.

22. Christmann RB, Wells AU, Capelozzi VL, Silver RM. Gastroesophageal reflux incites interstitial lung disease in systemic sclerosis: clinical, radiologic, histopathologic, and treatment evidence. Semin Arthritis Rheum 2010;40:241-9.

23. Lee JS, Collard HR, Anstrom KJ, et al. Anti-acid treatment and disease progression in idiopathic pulmonary fibrosis: an analysis of data from three randomised controlled trials. Lancet Respir Med 2013;1:369-76.

24. Adamek RJ, Wegener M, Wienbeck M, Pulte T. Esophageal motility disorders and their coexistence with pathologic acid reflux in patients with noncardiac chest pain. Scand J Gastroenterol 1995;30:833-8. 